\title{
Les lignes de désir de Chloé Savoie-Bernard et de Marilou Craft : féminisme rabat-joie et travail de la diversité dans la littérature québécoise
}

\author{
Joëlle Papillon \\ Université McMaster
}

Dans «Une féministe au carnaval», publié dans La Théorie, un dimanche (1988), l'écrivaine anglo-montréalaise Gail Scott réfléchit entre autres au mariage pas si simple entre féminisme et écriture. Pour mettre en lumière ce qu'elle considère être un problème, elle dédouble sa posture d'autrice en «la féministe » et «l'écrivaine », deux figures aux désirs différents : la féministe souhaite la création de modèles positifs (des femmes puissantes), tandis que l'écrivaine tient à sa liberté d'explorer l'humaine dans la lumière 
comme dans la noirceur (p. 50). Bien que parcourir les zones d'ombre lui apparaisse un danger en contexte féministe, l'écrivaine de Scott se décrit comme « [p] oussée par son désir d'aller au-delà de l'image presque trop parfaite de la "femme forte" d'une certaine fiction féministe », et souhaite plutôt «explorer les ténèbres » (p. 51, 52). À mon sens, nombre d'autrices québécoises contemporaines - de Nelly Arcan à Pattie O'Green, en passant par Audrée Wilhelmy et Marie Darsigny - suivent ce chemin « risqué », qu’elles se réclament ou non du féminisme, et proposent des personnages féminins ambigus, malmenés, souffrants, défaits, « tragiques ».

Dans le cadre de cet article, j'analyserai l'œuvre de deux écrivaines féministes de la relève qui, répondant à l'appel de Scott une trentaine d'années plus tard, nous offrent des « héroïnes ${ }^{1} »$ qui explorent le sombre et se présentent en pièces détachées. En résistance à l'invisibilisation persistante du travail des femmes racisées, j'ai voulu mettre en valeur l'œuvre de deux autrices noires montréalaises², Chloé Savoie-Bernard et Marilou Craft. Toutes deux mettent en scène des jeunes femmes qui, devant se définir dans un milieu plutôt hostile aux femmes - et particulièrement aux femmes noires -, développent des stratégies pour contester et détruire les normes sexistes et racistes qui leur assignaient une

\footnotetext{
${ }^{1}$ Il s'agit du terme employé par Scott dans « Une féministe au carnaval », ainsi que dans son roman Heroine (1987) paru plus ou moins en même temps que La Théorie, un dimanche. Le mot insiste sur la centralité du personnage féminin dans sa propre histoire, sa propre expérience du monde.

${ }^{2}$ Je tiens à reconnaître qu'en tant que femme blanche je n'ai pas été exposée personnellement à l'expérience violente du racisme. La réflexion de Peggy McIntosh (1988) sur le privilège blanc est toujours éminemment actuelle, et m'a aidée à réfléchir à ma positionnalité; on peut en consulter une adaptation française sur le site État d'exception (2017). URL: https://www.etatdexception.net/peggy-mcintosh-quest-ce-que-le-privilegeblanc/. Les deux autrices dont j'étudie les œuvres dans cet article n'ont pas bénéficié d'un tel privilège et évoquent diversement le rôle de la race dans leur expérience du monde. Quarante ans plus tard, les mots d'Audre Lorde résonnent toujours aussi fort lorsqu'elle critique le mutisme des féministes blanches en réfutant l'argument selon lequel celles-ci ne seraient pas directement concernées par la situation des femmes noires. Selon l'écrivaine afro-américaine, les féministes blanches tendent à se cacher derrière leur ignorance des œuvres et des expériences des femmes noires pour se désengager, ce qui a pour effet d'accentuer la marginalisation de celles-ci. Lorde nous exhorte à nous informer et à nous engager (2007 [1979], p. 113), ce que j'ai cherché à faire ici.
} 
JOËLLE PAPILLON, «Les lignes de désir de Chloé Savoie-Bernard et de Marilou Craft : féminisme rabat-joie et travail de la diversité dans la littérature québécoise »

place muette et invisible. Leurs œuvres tracent des «lignes de désir ${ }^{3} »$ ouvrant des chemins de traverse hors des sentiers habituels : et si on plaçait les œuvres de femmes racisées au centre de la littérature québécoise actuelle et non dans ses marges, quel portrait en obtiendrait-on? Mais avant de découvrir leurs textes, il convient de présenter succinctement la pensée de Sara Ahmed, qui a accompagné ma propre réflexion.

\section{Tuer la joie des autres}

Ahmed est une féministe queer de couleur basée à Londres, qui réfléchit d'un point de vue intersectionnel au sexisme, au racisme et à l'homophobie. Au fil de son œuvre, elle a développé une figure marquante, la feminist killjoy, qu'on a parfois traduite par « féministe rabat-joie $^{4} \gg$. Dans Living a Feminist Life (2017), Ahmed explique que cette figure part d'une accusation antiféministe; elle évoque un souper familial où tout le monde parle de façon appréciative du film Kramer vs. Kramer alors qu'elle s'inquiète que le film diabolise le personnage de la mère ${ }^{5}$. Immédiatement, tous se retournent contre elle et l'accusent d'avoir encore gâché un repas de famille avec son féminisme rabat-joie (p. 39). Ahmed investit cette figure en y reconnaissant une posture féministe : interrompre des joies patriarcales pour faire apparaitre comment elles sont problématiques et blessantes, comment elles soutiennent insidieusement la société hétérosexiste et raciste qui est la nôtre.

\footnotetext{
${ }^{3}$ Je reprends l'expression desire lines de Sara Ahmed (2017, p. 15), qui elle-même l'emprunte au champ de l'urbanisme; Ahmed l'utilise pour désigner les pistes qu'elle souhaite dégager à l'aide d'ouvrages théoriques féministes et antiracistes qui nous engagent sur des chemins inédits.

${ }^{4}$ C'est le terme retenu par Madeleine Stratford pour sa traduction de Notes From a Feminist Killjoy d'Erin Wunker (2016), paru en 2018 sous le titre Carnets d'une féministe rabat-joie aux Presses de l'Université de Montréal. Wunker s'inspire fortement d'Ahmed.

${ }^{5}$ Kramer vs. Kramer est un film réalisé par Robert Benton en 1979, qui relate le divorce entre Ted (Dustin Hoffman) et Joanna Kramer (Meryl Streep), et l'effet de celui-ci sur leur jeune fils Billy (Justin Henry). Les spectateurs et spectatrices sont amenés à éprouver de l'empathie pour Ted et Billy, tandis que Joanna est présentée comme égoïste.
} 
Parce que la féministe rabat-joie provoque une interruption (d'un souper de famille, d'une réunion, d'un discours, d'une conversation), elle bloque le cours habituel des choses et devient un irritant. Ahmed affirme qu'agir en tant que féministe rabat-joie tend à nous isoler (de notre famille, de nos collègues, de nos ami.es) puisqu'en faisant voir le racisme ou le sexisme d'une situation, la féministe rabat-joie en vient à avoir l'air de créer le problème :

[W] hen you name something as sexist or racist you are making that thing more tangible so that it can be more easily communicated to others. But for those who do not have a sense of the racism or sexism you are talking about, to bring them up is to bring them into existence. When you expose a problem you pose a problem ${ }^{6}$. (p. 3637; en gras dans l'original)

Exposer un problème, c'est poser un problème. Or, en explorant les zones d'ombre de leurs «héroïnes », Savoie-Bernard et Craft rejoignent toutes deux cette figure de la féministe rabat-joie, faisant apparaître les normes de genre et de race et à quel coût celles-ci se perpétuent.

\section{Chloé Savoie-Bernard : des ruines aux royaumes}

Royaume scotch tape (2015), premier recueil de poésie de Chloé Savoie-Bernard, dévoile plusieurs préoccupations notamment en lien à l'avortement et à la non-maternité, mais aussi au désir, à l'amitié féminine, et à la construction de soi à l'intersection du genre et de la race. Dans «ma face play-doh» (p. 52-53), la poète illustre la malléabilité de son corps, capable de contenir toutes les femmes noires - du moins dans le regard blanc posé sur elle. Par exemple, deux hommes l'arrêtent à différents moments en s'adressant à elle en anglais

\footnotetext{
${ }^{6}$ «Lorsque vous identifiez quelque chose comme sexiste ou raciste, vous rendez cette chose plus tangible afin de pouvoir la communiquer plus aisément aux autres. Mais pour ceux et celles qui ne sont pas sensibles au racisme ou au sexisme dont vous parlez, aborder ces questions les fait advenir. Quand vous exposez un problème, vous posez un problème. » (Je traduis)
} 
JOËLLE PAPILLON, «Les lignes de désir de Chloé Savoie-Bernard et de Marilou Craft : féminisme rabat-joie et travail de la diversité dans la littérature québécoise »

et en utilisant le nom d'une femme noire célèbre : « hey macy gray [...] / with your yellow coat your curly hair / you are the twin / $[\ldots]$ of that actress $[\ldots] » ; \ll[\ldots]$ hey are you rihanna's sister / or something ${ }^{7} »$ (p. 52, 53; minuscules dans l'original). La femme noire individuelle est noyée dans une série de visages possibles : comment distinguer Macy Gray de Rihanna ou de Chloé Savoie-Bernard, si tout ce qu'on voit est une image de femme noire générique? La fin du poème laisse bien sentir la douleur ressentie par la femme-reflet, visible en tant que femme noire mais invisible en tant qu'individu, qui disparaît en renvoyant à d'autres : « je suis cela / ce qui s'éteint dans mon visage / lorsqu'il réverbère / d'autres visages » (p. 53). De plus, la femme racisée se trouve interpellée en anglais, ce qui surdétermine sa position d'extériorité par rapport au groupe majoritaire. Dans ce poème, la féministe rabat-joie expose la persistance du racisme ordinaire au Québec ${ }^{8}$ et inscrit la souffrance de celles qui doivent en porter les effets.

Savoie-Bernard investit la figure de la féministe rabat-joie en faisant voir aux dépens de qui la société s'élève et se maintient. Dans Royaume scotch tape, plusieurs structures s'écroulent; c'est d'ailleurs sur ce mouvement de chute que le recueil s'ouvre, avec le poème «à l'agent d'immeuble »: «on ne l'achètera pas votre maison / l'expert a dit que les fondations étaient atteintes / gangrenées / [...] il faudrait tout reconstruire bientôt // on ne prendra pas ce risque-là » (p. 9). Plutôt que de s'investir dans le travail colossal de colmater fissure après fissure, la locutrice laisse les structures brisées se désintégrer; elle ne s'épuisera pas à essayer de sauver l'insauvable. Dans le poème suivant, elle affirme : «[...]

\footnotetext{
${ }^{7}$ « hey macy gray / avec ton manteau jaune tes cheveux frisés / tu es la jumelle / de cette actrice »; « hey es-tu la sœur de rihanna / ou quelque chose ». (Je traduis)

${ }^{8}$ Sur la question de la race dans la littérature québécoise, consulter De Groulx à Laferrière : Un parcours de la race dans la littérature québécoise de Corrie Scott (2014).
} 
pour que vous n'ayez pas raison de moi // oui me faire sorcière pour construire / mon propre royaume et en découdre avec le vôtre » (p. 11). Pourquoi réparer la maison de l'autre alors qu'elle peut aussi bien construire son propre royaume? Cette histoire de maison qui s'écroule évoque la phrase célèbre de l'autrice lesbienne afro-américaine Audre Lorde : « the Master's tools will never dismantle the Master's house ${ }^{9} »(1979)$, les outils du maître ne démantèleront jamais la maison du maître. Au moins depuis les années 1970, la métaphore de la société patriarcale, raciste et sexiste, en tant que maison à abattre, maison qui s'effondre, circule dans la littérature féministe et dans la littérature féministe noire en particulier. Laisser cette maison s'écrouler, voire l'aider à s'écrouler plus vite, semble l'une des tâches importantes de la féministe rabat-joie d'Ahmed qui nous enjoint à résister en s'écriant : « do not become the master's tool! » (p. 160), ne devenez pas l'outil du maître! Dans un autre poème de Royaume scotch tape, Savoie-Bernard présente des sujets vulnérables qui sont violemment jetés hors d'une maison :

ils détruiront encore nos maisons de paille et de bois ils souffleront dessus y mettront des mites des ogives des coquerelles nous ne nous réfugierons nulle part tant pis pour la foudre le feu le souffle des loups les demeures de pierre c'est pour les chickens

nous nous devons de voir à travers les murs nous ne craignons pas la dévoration (p. 14).

Persécuté par un ils sans référent, un nous non-assigné affirme sa force et sa résilience en dépit de sa position de fragilité; si « les demeures de pierre c'est pour les chickens », c'est

\footnotetext{
${ }^{9}$ Il s'agit du titre d'une présentation prononcée dans le cadre d'une conférence, puis publiée dans Sister Outsider: Essays and Speeches (2007 [1984]). Lorde s'y attaque particulièrement à l'absence de prise en compte sérieuse des différences entre femmes dans le cadre des réflexions féministes et à la marginalisation des intérêts des femmes noires et autres femmes en situation de minorité. Selon Lorde, en négligeant les femmes non-blanches, les féministes blanches reproduisent une dynamique patriarcale oppressive qu'elles tentent pourtant d'abolir.
} 
JOËLLE PAPILLON, «Les lignes de désir de Chloé Savoie-Bernard et de Marilou Craft : féminisme rabat-joie et travail de la diversité dans la littérature québécoise »

peut-être que le nous a toujours eu conscience de sa position précaire dans la maison, toujours susceptible d'en être évacué et de devoir faire face au loup.

Chez Savoie-Bernard, les maisons s'écroulent mais des royaumes s'élèvent. Si la locutrice se construit un royaume, c'est pour en être la reine, affirmer sa propre valeur et se placer au centre de cet autre monde plutôt qu'en périphérie. Plusieurs poèmes de Royaume scotch tape réfléchissent à l'autonomie : la locutrice et ses amies veulent se définir ellesmêmes, indépendamment du regard que leurs parents ou leurs amants posent sur elles. C'est dans ce cadre que le motif du royaume s'installe, de poème en poème; pour la locutrice, la liberté c'est être « reine de mon propre royaume sans sujet » (p. 19), c'est-àdire échapper aux maisons construites par les autres. L'expression « royaume scotch tape » surgit dans un poème relatant la relation houleuse entre une mère et sa fille adolescente. La locutrice décrit la façon dont elle a recouvert les murs de sa chambre de créatures monstrueuses créées en découpant des magazines : «j'avais fait des collages kinky / la tête de david bowie / sur le corps de marie-chantal toupin / des fleurs hawaïennes en background »(p.62; minuscules dans l'original). Le royaume scotch tape se présente comme un bricolage à partir de matériaux pas particulièrement nobles - des magazines invendus quêtés au dépanneur du coin - qui sont assemblés de façon à créer de l'imprévu. La jeune femme ne rejette pas ces matériaux ultra-normés (des publications qui font circuler l'image de corps de stars et qui participent à l'instauration et au maintien de normes de beauté qui favorisent la blanchitude ${ }^{10}$ ); au contraire, elle les reprend, les

\footnotetext{
${ }^{10}$ Les néologismes «blanchitude » ou «blanchité » ont été adoptés par les chercheur.es antiracistes pour désigner le fait d'être Blanc.he dans une société organisée sur le principe implicite ou explicite de la suprématie blanche. Alors que blancheur décrit une couleur, blanchitude renvoie spécifiquement aux privilèges associés à la peau blanche ou passant pour blanche. Voir par exemple Corrie Scott $(2014$, p. 26).
} 
transforme, les queere ${ }^{11}$ dans un geste créatif. La locutrice décrit son royaume avec humour, mais on sent qu'elle en est fière : «mon royaume scotch tape / n'est pas ton royaume »(p.62), dit-elle à sa mère, puisqu'à travers ses collages elle est parvenue à redéfinir la chambre qu'elle occupait dans la maison de sa mère en royaume qui lui ressemble. À l'image des écrivaines suicidées qu'elle admire dans un autre poème (p. 4851), elle aussi est une perle irrégulière.

Le scotch tape évoque la fragilité d'une construction temporaire, les fragments menaçant toujours de s'éparpiller à nouveau. C'est que la locutrice de Savoie-Bernard tient toujours à peine ensemble, est toujours sur le point de se désarticuler. Dans le second recueil de la poète, Fastes (2018), le morcellement du corps féminin est encore plus évident : la locutrice, « déprise de [s]on unité » se présente «morceau par morceau » (p. 11) et est « à la recherche de ce qui [la] découd» (p. 15). Elle raconte comment, au réveil, elle doit s'activer au « rapatriement » des morceaux : « [...] tout se met à peu près en place // à peu près comme il faut » (p. 20, 21). Même rafistolé, on sent bien que le corps de la locutrice demeure fissuré, le scotch tape bien apparent aux coutures. Dans les deux recueils, on retrouve ce jeu constant entre dépeçage et rapiéçage en lien avec le corps féminin ${ }^{12}$; devant ces corps de femmes qui se désagrègent, une responsabilité nous échoit, celle de prendre $\operatorname{soin}^{13}$ : danger, manipulez avec soin.

L'un des plus beaux poèmes de Royaume scotch tape, «prévision météorologique », présente le corps des filles comme un lieu de blessure et de désir : «samedi soir une fois

\footnotetext{
${ }^{11}$ Dans le contexte des études queer, on utilise fréquemment le verbe to queer pour désigner un geste qui crée de l'imprévu ou du bizarre, faisant apparaître du même coup la norme et des voies d'échappement.

${ }^{12}$ Il ne paraît pas indifférent d'ailleurs que, dans ses travaux de chercheure, Savoie-Bernard (2018b) se soit intéressée à la stratégie du collage dans les œuvres féministes des années 1970 : découper, coller, créer.

${ }^{13}$ Sara Ahmed écrit : « fragility gives us responsibility : to take care » $(2017$, p. 17).
} 
JOËLLE PAPILLON, «Les lignes de désir de Chloé Savoie-Bernard et de Marilou Craft : féminisme rabat-joie et travail de la diversité dans la littérature québécoise »

encore / des filles fumées jusqu'au filtre / des filles fleurs en manque de pollen / qui s'étiolent pétale après pétale / des filles tomberont des fenêtres / crachin dans villeray mile end hochelaga »(p.22; minuscules dans l'original). La poète investit des figures de femmes brisées mais toujours en train de se relever, résilientes, intuables, puisque la fin de «prévision météorologique » voit les filles s'épousseter et rejoindre leurs amants malgré leurs jambes tordues. Ce faisant, Savoie-Bernard parvient à réconcilier les impératifs contraires que Gail Scott avait identifiés : représenter des femmes puissantes tout en explorant la dimension «tragique » de la féminité en contexte patriarcal (p. 50). Le dernier poème du recueil, « où vont les filles comme nous », montre un groupe de filles qui avancent dans la ville telles des bulldozers, fonçant sur des obstacles sans s'arrêter; le poème se clôt ainsi : «[...] nous y allons avec tout notre allant / nous nous pognons des murs dans la gueule / nous les traversons quand même // avoir du plâtre dans la bouche / c'est toujours mieux / que de ne rien manger du tout » (p. 74). La ville comme la maison est en ruines, mais les filles vivent, elles marchent, elles se recoudront l'une l'autre. Les fillesbulldozers font foi de la force et de la résilience féminines, sans masquer la souffrance, le coût élevé de la survie des filles et des femmes dans cette ville inhospitalière.

Dans l'œuvre de Savoie-Bernard, la violence du monde sexiste et raciste se lit à même le corps des femmes et des filles couvert de cicatrices, d'autres marques des coutures qui tiennent les morceaux en place. À mon sens, la fragilité de l'assemblage qui se maintient avec du scotch tape peut paradoxalement signaler une force particulière : celle de la capacité de se reconstruire, de se réassembler, de se réinventer à l'infini. Comme la locutrice l'affirmait dans un poème cité plus haut, les sujets en position précaire sont particulièrement sensibles et inventifs : «nous nous devons de voir à travers les murs » 
(p. 14). De la même façon qu'elle ne pleurera pas la maison effondrée parce qu'elle peut toujours créer son propre royaume, la locutrice de Savoie-Bernard ne s'attristera pas de son corps accidenté puisque chaque blessure est l'occasion de constater qu'elle ne peut être anéantie. D'une certaine façon, en contexte patriarcal, le corps féminin peut lui aussi être considéré comme « la maison du maître », une maison dont on doit exposer les fissures avant de la recomposer de façon «kinky» avec nos propres outils et beaucoup de scotch tape. Dans l'œuvre de la poète, fragilité et force ne sont pas des contraires mais s'appuient l'une sur l'autre. En explorant le sombre, Savoie-Bernard illumine un autre genre de femmes puissantes qui, loin de nier le côté «tragique » du féminin, y trouvent un certain plaisir et une source de pouvoir.

\section{La féministe rabat-joie et le travail de la diversité}

Sara Ahmed consacre l'une des trois parties de Living a Feminist Life à ce qu'elle nomme « diversity work », le travail de la diversité. En se basant sur son expérience personnelle dans le milieu universitaire, Ahmed étudie notamment les efforts qu'elle-même et d'autres « diversity workers » (travailleuses et travailleurs de la diversité) ont déployés pour transformer leur institution en profondeur. Elle démontre comment, en dépit du discours prônant la diversité et l'égalité, les universités demeurent toujours un milieu structuré par le racisme qui privilégie les hommes blancs (p. 90-91). Par conséquent, les femmes de couleur qui travaillent au sein des universités occupent une position d'ethnographes (p. 91), d'observatrices critiques, et participent - qu'elles le veuillent ou non - au travail de la diversité. Ahmed définit ce travail de la façon suivante : «I use diversity work in two related senses : first, diversity work is the work we do when we are attempting to transform 
JOËLLE PAPILLON, «Les lignes de désir de Chloé Savoie-Bernard et de Marilou Craft : féminisme rabat-joie et travail de la diversité dans la littérature québécoise »

an institution; and second, diversity work is the work we do when we do not quite inhabit the norms of an institution ${ }^{14}$.» (p. 91)

Ahmed insiste à plusieurs reprises sur la difficulté de ce travail, sur l'effort pénible de porter le travail de la diversité à même son corps, qui est tour à tour invisible et trop visible. Elle relate par exemple que les femmes de couleur ne sont souvent pas reconnues comme des sujets intellectuels au même titre que leurs collègues (invisibilité), mais que leur présence est fréquemment perçue comme une irruption problématique (visibilité) (p. 126128). L'une des images récurrentes dans l'ouvrage d'Ahmed est celle du mur de briques contre lequel la tête de la féministe rabat-joie, la tête de la travailleuse de la diversité viennent se cogner à répétition (p. 110), ce qui évoque les autres murs (de plâtre) contre lesquels les filles se fracassaient dans le poème de Savoie-Bernard cité plus haut. Avoir un corps perçu comme différent - voire incongru - constitue donc une assignation à un travail ardu, toujours à recommencer. Cette question traverse la nouvelle « $\mathrm{A} / \mathrm{S} / \mathrm{V} »$ de Marilou Craft - parue dans le recueil Corps (2018) dirigé par Chloé Savoie-Bernard - qui montre comment le simple fait d'être une femme noire dans un milieu majoritairement blanc occasionne un travail de la diversité douloureux.

\section{Marilou Craft, ou le corps inadéquat}

Craft, qui publie à l'occasion des chroniques, a été parmi celles et ceux qui sont intervenus dans le débat autour de la pièce $S L \bar{A} V$ de Betty Bonifassi et Robert Lepage, à l'affiche au TNM lors de la saison 2017-2018 (Craft, 2017; 2018b). En tant que féministe noire, Craft a souligné les problèmes que posait la conception de la pièce, créée à partir de chants d'Afro-

\footnotetext{
14 «J'utilise le travail de la diversité de deux façons interreliées : d'abord, le travail de la diversité est le travail qu'on fait lorsqu'on cherche à transformer une institution; ensuite, le travail de la diversité est le travail qu'on fait lorsqu'on ne se conforme pas tout à fait aux normes d'une institution. » (Je traduis)
} 
Américains réduits en esclavage par une équipe presque entièrement blanche. En soulevant les questions de l'appropriation culturelle et de l'effacement des personnes noires et de la spécificité de leurs expériences, Craft s'est interposée en tant que féministe rabat-joie, « gâchant le plaisir » des spectateurs et de ceux et celles qui, comme Bonifassi, « ne voient pas la couleur » et croient que le racisme est chose du passé au Québec. Parce qu'exposer un problème, c'est poser un problème et que le travail de la diversité amène à se fracasser contre un mur (qu'il soit de briques ou de plâtre), Craft a fait l'objet d'une campagne de salissage violente. C'est dans ce contexte de visibilité pénible que sa nouvelle « $\mathrm{A} / \mathrm{S} / \mathrm{V} »$ est parue.

La nouvelle est composée de deux fils de fragments entrecroisés : dans le premier fil, on retrouve «Marilou » à des moments marquants de sa vie, de 1 à 30 ans; dans le second, des hommes prennent la parole sur des médias sociaux ou des sites de rencontre pour commenter l'apparence et la désirabilité de Marilou, en lien à sa race perçue. « $\mathrm{A} / \mathrm{S} / \mathrm{V}$ » illustre parfaitement comment le racisme et le sexisme sont chevillés : l'un n'arrive pas avant l'autre, l'un n'est pas plus blessant que l'autre, ils fonctionnent ensemble dans la vie de la protagoniste. C'est pour aborder ce nœud des oppressions que des féministes afroaméricaines telles que Kimberlé Crenshaw et Patricia Hill Collins ont développé la notion d'intersectionnalité $^{15}$ : puisque les femmes noires se trouvent à l'intersection du racisme et du sexisme (les exposant à ce que Moya Bailey appelle la «misogynoir » ${ }^{16}$ ), il est

\footnotetext{
${ }^{15}$ En français, voir notamment Crenshaw (2005 [1991]) et Hill Collins (2016 [2009]). Consulter aussi l'ouvrage que Patricia Hill Collins et Sirma Bilge (2016) ont co-écrit pour définir ce concept.

${ }^{16}$ Moya Bailey est une féministe afro-américaine queer; elle a créé le terme « misogynoir » pour désigner le visage unique de la violence à laquelle les femmes noires sont confrontées dans la culture américaine (2018, p. 763). La blogueuse afro-américaine Trudy, qui a participé au développement de la notion, souligne que misogynoir permet de centrer l'expérience des femmes noires (2018, p. 764).
} 
JOËLLE PAPILLON, «Les lignes de désir de Chloé Savoie-Bernard et de Marilou Craft : féminisme rabat-joie et travail de la diversité dans la littérature québécoise »

nécessaire de tenir compte de ces deux facteurs pour comprendre leur situation et combattre les iniquités qui en découlent. Dans sa nouvelle, Craft montre bien que le racisme suit Marilou toute sa vie, puisque le premier fragment relate une scène de rejet violent causé par la perception de son corps comme inadéquat, alors qu'elle n'a qu'un an. Selon l'éducatrice de la garderie où sa mère l'a déposée, Marilou pleure constamment parce qu'« [e]lle est laide et elle pue » (p. 56). Avec ces mots cruels, l'éducatrice signale que le corps de l'enfant est un affront, et sous-entend qu'elle porte une déficience interne : si elle est triste, si elle a mal, ce serait de sa propre faute. Pour protéger sa fille du racisme, la mère de Marilou la retire de la garderie, mais le sentiment d'inadéquation et la douleur de l'enfant persistent, puisqu'elle conclut : «Je pleure pareil » (p. 56). Ce premier fil de fragments se développe selon deux axes interreliés : l'expérience du racisme anti-noir, et le sentiment d'avoir un corps inadéquat et repoussant.

À l'âge de onze ans, lors d'une fête d'enfants chez une amie blanche, Valérie, Marilou découvre avec stupeur que les poils sur les jambes des filles sont laids et honteux. Elle surprend une conversation entre la mère de Valérie et une de ses amies, où elles sont d'accord qu'il n'est jamais trop tôt pour commencer l'épilation des filles, qu'il faut dès maintenant commencer à les habituer au rituel douloureux de la cire épilatoire afin de les désensibiliser (p. 58). Marilou observe avec consternation les longs poils noirs qui collent à ses propres jambes au sortir de la piscine; elle cherche son amie Valérie du regard, puis voit les jambes de celle-ci qui se dressent hors de l'eau : «Sa peau est éclatante, lumineuse, lisse. Une chandelle parfaite. » (p. 59) La chandelle parfaite réussie par Valérie est à la fois jeu enfantin et performance de la féminité blanche hétéronormative : seules les jambes parfaitement épilées peuvent s'afficher fièrement de la sorte, dans une sorte de triomphe 
que Marilou n'atteindra jamais, elle qui adaptera sa garde-robe au besoin de cacher ses jambes en tout temps durant l'adolescence.

À treize ans, ce seront encore les poils noirs qui viendront l'humilier dans une scène particulièrement douloureuse où Louis, le garçon qu'elle aime, la traitera de « grizzly » en riant (p. 61). Craft insiste sur la blanchitude de ce garçon :

Je regarde Louis encore et encore, ses yeux bleus, sa peau blanche. Je regarde la peau blanche de Louis alors qu'il se penche pour écouter ce que Mathieu lui chuchote à l'oreille. Je regarde les yeux bleus de Louis pétiller [...]. Je regarde les yeux bleus de Louis me regarder, descendre, descendre, regarder mes jambes, fixer mes jambes. Je regarde Louis rire avec Mathieu, lumineux, lumineux (p. 61).

Louis et Mathieu, avec leur peau et leurs yeux « lumineux »-comme l'étaient les jambes de Valérie - occupent une position privilégiée d'où ils peuvent observer, juger et railler la fille-grizzly, qui reste dans l'ombre. Marilou a déjà internalisé et accepté leur droit de la traiter de cette façon, puisque le fragment se termine sur le petit bonheur que Louis lui ait adressé la parole malgré tout. Dans les fragments subséquents de ce fil, on suivra la bataille acharnée de Marilou contre ses poils disgracieux, dans le double contexte de son image de soi et de ses relations érotiques.

Tandis que ces fragments sont organisés de façon chronologique, ceux du deuxième fil ne sont pas datés et pourraient aussi bien se rapporter tous au présent ou s'étaler sur une période beaucoup plus longue. Ils sont constitués de discours attribués à des hommes identifiés par un prénom et un âge, et prennent l'apparence de messages échangés dans le cadre de flirt en ligne. Le fait qu'ils soient intercalés dans le fil autobiographique a pour effet de suggérer que les commentaires misogynoirs ont accompagné Marilou tout au long de sa vie. Pour la plupart, ces hommes affichent un désir d'elle, mais celui-ci s'exprime d'une façon qui la diminue - que ce soit en saluant son apparence exotique, en lui 
JOËLLE PAPILLON, «Les lignes de désir de Chloé Savoie-Bernard et de Marilou Craft : féminisme rabat-joie et travail de la diversité dans la littérature québécoise »

demandant à répétition d'où elle vient, en vantant le sexy des femmes africaines, ou en s'excitant à l'idée de faire l'amour avec une femme noire pour la première fois. D'autres, moins nombreux, signalent que le fait qu'elle soit noire est un empêchement au désir, ce qui ne serait «pas de leur faute». Dans tous les cas, Marilou est fermement placée à l'intersection du genre et de la race, à un endroit où elle n'est rien en tant qu'individu - qui serait comme ceci ou comme cela -, où elle ne joue que le rôle d'une femme noire - de la femme noire -, remplaçable à l'infini, ce qui n'est pas sans rappeler la situation décrite par Savoie-Bernard dans « ma face play-doh ».

Dans ce fil de fragments, la narratrice de Craft fait l'expérience douloureuse du «racisme sexuel», un phénomène qui touche tout particulièrement les femmes noires (Bedi, 2015, p. 998) et qui s'exprime tout autant dans le fétichisme que dans le rejet de partenaires racisé.es (Gibert, 2016, p. 143). Le politicologue Sonu Bedi définit le « racisme sexuel » comme le fait de choisir un ou une partenaire sexuel.le en fonction de sa race perçue, de manière à soutenir les stéréotypes raciaux ou la hiérarchie raciale en place dans une société donnée (p. 998). Selon lui, le domaine des relations intimes n'est pas à l'abri des enjeux d'équité, puisque l'opportunité de développer des relations intimes est un «bien social primaire » (a social primary good) et est essentielle à la dignité humaine (p. 998). En outre, Bedi réfute l'argument que l'attirance ou la non-attirance envers des personnes de telle ou telle race perçue puisse n'être qu'une affaire de goût personnel : en regard du racisme passé et actuel qui traverse toutes les sphères de l'existence (l'accès au travail et au logement, le sentiment de sécurité et le rapport aux autorités, etc.), la race est un axe marqué comme aucun autre. Pour Bedi, être jugé.e plus ou moins attirant.e en fonction de notre appartenance raciale renforce les stéréotypes et les normes raciales, réinscrivant les 
individus comme ayant plus ou moins de valeur selon la place qu'on leur attribue sur le spectre racial (p. 1004).

Dans « $\mathrm{A} / \mathrm{S} / \mathrm{V} »$, Craft présente de façon pêle-mêle et sans commentaire les messages des hommes du Net qui l'évaluent; toutefois, sa critique féministe antiraciste est clairement audible en dépit de son absence textuelle. Dans l'accumulation de ces commentaires désobligeants - tous différents mais d'une certaine façon tous pareils - se dessine la longue tirade misogynoire qui attaque Marilou au quotidien. Dans certaines entrées plus longues, on voit que Marilou a répondu à ses interlocuteurs, ce qu'elle indique par des crochets d'ellipse, et qu'elle les a confrontés au racisme de leur propos. Leurs répliques défensives font bien voir que la féministe rabat-joie a encore tout gâché : c'était «seulement un compliment »(p. 76), «je voulais pas toffencer... mais jaime quand meme lexotisme » (p. 79; sic), «Bah c'est une préférence un gout rien d'autre » (p. 82), «Bah je vois pas ou est le mal en fait?» (p. 82; sic), « on s'en fout tellement de la couleur la, sérieux... Relax » (p. 83; sic). Dans tous ces fragments, les hommes du Net ne comprennent aucunement la façon dont la narratrice reçoit les mots qu'ils lui envoient; leur ton est résolument badin et léger, leurs messages ponctués d'emojis souriants ou taquins, d'expressions telles que «lol» ou « haha », mais Marilou ne rit pas. Elle est consciente que, comme dans l'épisode de la fille-grizzly, la blague est faite à ses dépens et à ceux des femmes comme elle. Adoptant la posture d'« ethnographe » décrite par Ahmed (p. 91), elle observe de façon critique et documente le rôle du facteur racial dans les relations de désir. En se prenant pour objet, l'ethnographe expose moins son expérience individuelle du monde que la façon dont 
JOËLLE PAPILLON, « Les lignes de désir de Chloé Savoie-Bernard et de Marilou Craft : féminisme rabat-joie et travail de la diversité dans la littérature québécoise »

le racisme structure l'existence des femmes racisées ${ }^{17}$. En tant que féministe rabat-joie, Craft fait «apparaître le racisme », la misogynoir, là où pour les personnes blanches - et particulièrement pour les hommes blancs du Net - il n’y avait rien : rien à voir, rien à dire, rien pour s'offusquer. En outre, Craft montre le pénible travail de la diversité effectué par Marilou à la fois activement lorsqu'elle tente d'éduquer les hommes du Net en leur expliquant en quoi leurs remarques sont racistes, et passivement du seul fait d'être un corps noir dans un océan de blanchitude.

\section{Le bonheur, quand ça?}

Comme le remarquait déjà Lori Saint-Martin dans Contre-voix en 1997 (p. 237), les autrices québécoises contemporaines sont fréquemment comparées à leurs aînées de façon assez négative : elles seraient moins engagées, moins radicales, moins politiques que les autrices féministes des années 1970. Pourtant, les nouvelles formes qu'emprunte le féminisme littéraire n'ont pas à être conçues comme un amoindrissement ou une perte : «le féminisme change, la fiction aussi. À nous, lectrices, lecteurs, de les suivre » (Saint-Martin, 1997, p. 268). L’une des routes que Chloé Savoie-Bernard et Marilou Craft nous invitent à prendre est celle de l'exploration critique de l'intersection genre/race : en quoi l'expérience des femmes québécoises racisées est-elle spécifique? Loin de concerner uniquement les femmes racisées, cette question requiert au contraire un investissement critique de toutes et tous.

Lors de sa réédition en 2018, Martine Delvaux signe la préface de La Théorie, un dimanche; elle y célèbre l'importance du legs féministe que l'ouvrage constitue, mais elle

\footnotetext{
${ }^{17}$ Commentant les projets du type Everyday Sexism qui documentent les micro-agressions, Ahmed affirme que ceux-ci permettent aux femmes de confirmer que leur expérience du sexisme et du racisme est largement partagée, constituant en fait la «structure » de l'expérience des femmes (p. 30).
} 
se tourne aussi vers aujourd'hui et vers demain en terminant sur un appel à la diversité. À la «table du féminisme» québécois actuel, Delvaux convoque «des héroïnes diverses, venues de partout, aux visages, à la peau, à la culture, aux désirs différents », qui portent un regard «kaléidoscopique » sur le monde (p. 17). Bien qu'il y ait eu une certaine diversité chez les auteures féministes québécoises des années 1970 et 1980, l'absence de diversité raciale est significative ${ }^{18}$. La notion de «travail de la diversité » proposée par Ahmed lorsqu'elle réfléchit aux universités me paraît aisément applicable au milieu littéraire, qui constitue également une institution que les travailleuses et travailleurs de la diversité doivent « ouvrir » à celles et ceux qui en ont été exclus ${ }^{19}$. Comme l'illustre Ahmed en ayant recours à la métaphore du «mur de briques », ce travail est difficile et souffrant, entre autres parce qu'il doit être sans cesse recommencé, qu'il expose la travailleuse de la diversité en l'associant au problème qu'elle tente de régler, et qu'il la place dans une position vulnérable où elle est moins supportée par l'institution (p. 96). La travailleuse de la diversité rejoint par là la figure de la féministe rabat-joie, ce qu'Ahmed souligne en lui octroyant le titre d'«institutional killjoy» (p.99), celle qui interrompt la joie de l'institution dans laquelle elle occupe une position marginale.

\footnotetext{
${ }^{18} \mathrm{Si}$ l'on peut parler d'une « certaine diversité », c'est pour souligner la visibilité des féministes lesbiennes à cette époque, ainsi que l'audibilité des revendications des féministes prolétaires - notamment dans le cadre d'entreprises de travail littéraire collectif lié à l'éveil des consciences politiques (Si Cendrillon pouvait mourir! [1975] et Môman travaille pas, a trop d'ouvrage! [1975], par exemple). En outre, une diversité linguistique se dessine modestement grâce à la participation de l'écrivaine anglophone Gail Scott à la scène littéraire féministe montréalaise. Si le milieu féministe québécois actuel est plus diversifié du point de vue racial que dans les années 1970 ou 1980, il reste un long chemin à faire pour atteindre une représentation adéquate des intérêts des femmes racisées. À ce sujet, consulter l'important ouvrage codirigé par Naïma Hamrouni et Chantal Maillé, Le sujet du féminisme est-il blanc? (2015).

${ }^{19}$ Je paraphrase Ahmed lorsqu'elle définit le travail de la diversité comme «the work we do when we are trying to open up institutions to those who have historically been excluded from them » (p. 93).
} 
JOËLLE PAPILLON, «Les lignes de désir de Chloé Savoie-Bernard et de Marilou Craft : féminisme rabat-joie et travail de la diversité dans la littérature québécoise »

À la lecture de Savoie-Bernard, de Craft et de leurs contemporaines (O'Green, Wilhelmy, Darsigny, mais aussi des écrivaines autochtones telles que Natasha Kanapé Fontaine ou Naomi Fontaine), on remarque aisément que la littérature des femmes actuelle prend rarement le chemin de la joie ou de la légèreté. Cela dit, l'expression d'autres affects peut avoir un effet tout aussi libérateur - sinon plus -, comme en fait foi le collectif Libérer la colère (2018), dans lequel la création d'un espace légitime où formuler la colère des femmes est lui-même perçu comme une source de joie (p. 16). On gagnerait à remettre en question nos attentes en ce qui concerne la littérature des femmes : pourquoi y cherche-t-on souvent l'expression d'un bonheur? Comme l'explique magnifiquement Ahmed dans The Promise of Happiness (2010), nombre de féministes choisissent de ne pas suivre le chemin du bonheur dans la mesure où ce dernier est généralement construit de façon étriquée comme la voie du mariage hétérosexuel et de la maternité. Pour Ahmed, «feminist consciousness [is] a form of unhappiness ${ }^{20} \gg$ (p. 53) parce que les féministes rabat-joie refusent de suivre le modèle qu'on nous présente - traditionnellement celui de la femme au foyer épanouie. Ahmed établit une archive d'expressions d'insatisfaction et de tristesse pour montrer comment celles-ci bâtissent une contestation féministe de nos sociétés patriarcales sexistes et racistes. À mon sens, Savoie-Bernard et Craft s'inscrivent parfaitement dans cette archive: elles expriment toutes deux moins une souffrance individuelle qu'une contestation profonde du script du bonheur (Ahmed, 2010, p. 59) dont elles ont hérité : est-on sur la voie du bonheur si on est une femme noire au Québec? Et, ce

\footnotetext{
${ }^{20}$ On pourrait traduire maladroitement par « la conscience féministe est une forme de non-bonheur », puisque $\mathrm{ni} \ll$ malheur $» \mathrm{ni}$ « tristesse » ne semblent adéquats pour traduire unhappiness ici.
} 
faisant, elles rejoignent leurs prédécesseures qui, rabat-joie elles aussi, souhaitaient déjà la mort de Cendrillon.

\section{Bibliographie}

AHMED, Sara (2017), Living a Feminist Life, Durham et Londres, Duke University Press.

AhMed, Sara (2010), The Promise of Happiness, Durham et Londres, Duke University Press.

BEDI, Sonu (2015), «Sexual Racism : Intimacy as a Matter of Justice », The Journal of Politics, vol. 77, n 4, p. 998-1011.

BAILEY, Moya et TRUDY (2018), «On Misogynoir : Citation, Erasure, and Plagiarism », Feminist Media Studies, vol. 18 n 4, p. 762-768.

CRAFT, Marilou (2017), «Qu'est-ce qui cloche... avec le prochain spectacle de Betty Bonifassi », Urbania, 5 décembre. URL : https://urbania.ca/article/quest-ce-qui-clocheavec-le-prochain-spectacle-de-betty-bonifassi/ (consulté le 11 janvier 2019)

CRAFT, Marilou (2018a), «A/S/V », dans Chloé Savoie-Bernard (dir.), Corps, Montréal, Triptyque, p. 55-85.

CRAFT, Marilou (2018b), «SLĀ V: Le bilan de Marilou Craft », Urbania, 3 juillet. URL : https://urbania.ca/article/slav-bilan-de-marilou-craft/ (consulté le 11 janvier 2019)

Crenshaw, Kimberlé W. (2005 [1991]), «Cartographies des marges : Intersectionnalité, politique de l'identité et violences contre les femmes de couleur », trad. Oristelle Bonis, Cahiers du Genre, n 39 , p. 51-82.

DelvauX, Martine (2018), «Tous les jours, dimanche », dans Louky Bersianik, Nicole Brossard, Louise Cotnoir, Louise Dupré, Gail Scott et France Théoret, La Théorie, un dimanche [1988], Montréal, les éditions du remue-ménage, p. 9-17.

GIBERT, Martin (2016), «L'étrange cas du racisme sexuel», Nouveau projet, $\mathrm{n}^{\circ}$ 9, printemps-été, p. 141-144.

HAMrouni, Naïma et Chantal MAILLÉ (dir.) (2015), Le sujet du féminisme est-il blanc? Femmes racisées et recherche féministe, Montréal, les éditions du remue-ménage. 
JOËLLE PAPILLON, « Les lignes de désir de Chloé Savoie-Bernard et de Marilou Craft : féminisme rabat-joie et travail de la diversité dans la littérature québécoise »

Hill collins, Patricia (2016 [2009]), La Pensée féministe noire : Savoir, conscience et politique de l'empowerment, trad. Diane Lamoureux, Montréal, les éditions du remueménage.

Hill COllins, Patricia et Sirma BILGE (2016), Intersectionality, Cambridge et Malden, Polity Press.

Le Show Des Femmes De ThetFord Mines (1980 [1975]), Si Cendrillon pouvait mourir!, Montréal, les éditions du remue-ménage.

LE ThÉÂtRe Des CuISINES (1976 [1975]), Môman travaille pas, a trop d'ouvrage!, Montréal, les éditions du remue-ménage.

LORDE, Audre (1979), «The Master's Tools Will Never Dismantle the Master's House », dans Sister Outsider: Essays and Speeches (2007 [1984]), Berkeley, Crossing Press, p. 110-113.

MCINTOSH, Peggy (2017 [1988]), « Privilège blanc, déballer le havresac invisible », traduit et adapté par LQP et RC, État d'exception. URL: https://www.etatdexception.net/peggymcintosh-quest-ce-que-le-privilege-blanc/ (consulté le 10 mars 2019)

MORAND, Geneviève et Natalie-Ann ROY (dir.) (2018), Libérer la colère, Montréal, les éditions du remue-ménage.

SAINT-MARTIN, Lori (1997), « Trois romans métaféministes », dans Contre-voix : Essais de critique au féminin, Montréal, Nuit blanche éditeur, coll. «Essais critiques », p. 235-268.

SAVOIE-Bernard, Chloé (2015), Royaume scotch tape, Montréal, L’Hexagone.

SAVOIE-Bernard, Chloé (2018a), Fastes, Montréal, L’Hexagone.

SAVOIE-BERnARD, Chloé (2018b), «Les femmes comme au musée. Communautés et temps suspendu dans la littérature contemporaine québécoise », dans Julie Beaulieu, Adrien Rannaud et Lori Saint-Martin (dir.), Génération(s) au féminin et nouvelles perspectives féministes, Québec, Codicille, coll. «Prégnance », p. 241-265.

SCOTT, Corrie (2014), De Groulx à Laferrière : Un parcours de la race dans la littérature québécoise, Montréal, XYZ, coll. « Théorie \& littérature ».

ScotT, Gail (1987), Heroine, Toronto, The Coach House Press.

ScotT, Gail (2018 [1988]), «Une féministe au carnaval», trad. Claudine Vivier, dans Louky Bersianik, Nicole Brossard, Louise Cotnoir, Louise Dupré, Gail Scott et France Théoret, La Théorie, un dimanche, Montréal, les éditions du remue-ménage, p. 49-84. 


\title{
Résumé
}

Cet article analyse l'œuvre de Chloé Savoie-Bernard et de Marilou Craft afin de délimiter l'une des voies que prend la relève féministe au Québec. Les deux autrices placent leurs sujets-femmes à l'intersection du genre et de la race et adoptent une posture de féministe rabat-joie (Sara Ahmed) pour dénoncer la position précaire des femmes racisées, notamment dans les relations intimes. Bien que les écrivaines de la relève semblent s'éloigner du modèle féministe des "femmes puissantes », on propose ici qu'en explorant les zones d'ombre de l'expérience de la féminité, Savoie-Bernard et Craft participent de façon innovante à la contestation des normes de genre et de race.

\begin{abstract}
This article studies works by Chloé Savoie-Bernard and Marilou Craft in order to delineate one of the paths embraced by the new generation of Québécois feminist writers. The two authors put their female subjects at the intersection of gender and race and adopt the stance of the feminist killjoy (Sara Ahmed) to denounce the precarious position of racialized women, most notably when it comes to intimate relationships. Although this new generation seems to leave behind the feminist model of "powerful women », this article argues that by exploring the darker sides of the experience of femininity, Savoie-Bernard and Craft contest gendered and racial norms in an innovative way.
\end{abstract}

\section{Notice biobibliographique}

Joëlle Papillon est professeure à l'Université McMaster, où elle enseigne les littératures autochtone, franco-canadienne et québécoise. Elle s'intéresse particulièrement à la littérature actuelle des femmes (Désir et insoumission chez Arcan, Millet et Ernaux, Presses de l'Université Laval, 2018; Nelly Arcan : Trajectoires fulgurantes [codir.], les éditions du remue-ménage, 2017) et des Premières Nations. Elle vit et travaille sur le territoire traditionnel et actuel des peuples Haudenosaunee et Anishinabé, qu'elle remercie pour leur hospitalité. 